\title{
Kinematical constraint effects in the evolution equations based on angular ordering
}

\author{
Michal Deak $^{a}$ and Krzysztof Kutak ${ }^{b}$ \\ ${ }^{a}$ IFIC, Universitat de València-CSIC, \\ Apt. Correus 22085, E-46071 València, Spain \\ ${ }^{b}$ Instytut Fizyki Jadrowej, Polskiej Akademii Nauk, \\ Radzikowskiego 152, 31-342 Krakow, Poland \\ E-mail: michal.deak@ific.uv.es, krzysztof.kutak@ifj.edu.pl
}

\begin{abstract}
We study effects of imposing various forms of the kinematical constraint on the full form of the CCFM equation and its non-linear extension. We find, that imposing the constraint in its complete form modifies significantly the shape of gluon density as compared to forms of the constraint used in numerical calculations and phenomenological applications. In particular the resulting gluon density is suppressed for large values of the hard scale related parameter and $k_{T}$ of gluon. This result might be important in description of jet correlations at Large Hadron Collider within the CCFM approach.
\end{abstract}

KeYwords: QCD Phenomenology, Deep Inelastic Scattering (Phenomenology)

ArXIV EPrint: 1503.00536v2 


\section{Contents}

1 Introduction 1

2 The CCFM equation $\quad 2$

2.1 The kinematical constraint 3

2.2 Saturation effects and kinematical constraint combined 5

3 Numerical results and the discussion $\quad 6$

$\begin{array}{lll}3.1 & \text { Linear equations } & 6\end{array}$

3.2 Non-linear equation $\quad 8$

$\begin{array}{llr}3.3 & \text { Dependence on the starting scale } & 9\end{array}$

$\begin{array}{lll}4 & \text { Conclusions and outlook } & 10\end{array}$

\section{Introduction}

In the high energy limit of hadron scattering, in the regime where the center of mass energy is larger than any other available scale, perturbative approach to processes with high momentum transfer allows factorization of the cross section into a hard matrix element with initial off-shell gluons and an unintegrated gluon density [1, 2]. The unintegrated gluon density is a function of the longitudinal momentum fraction $x$ and transverse momentum $k_{T}$ of a gluon. After taking into account formally subleading corrections coming from coherence of gluon emissions, one is lead to the CCFM set of equations [3-5] which introduce gluon density dependent on hard scale related to the probe as well as one introduces unintegrated quark densities. The status of phenomenological relevance of CCFM framework is not fully satisfactory. In principle, it is a set of equations that should be the ideal framework for application to final states at high energies covering DGLAP and BFKL domains. It has been implemented in the Monte Carlo event generator [6]. However, so far good agreement with high precision data has been successfully achieved only in rather inclusive processes like $F_{2}$ and Drell-Yan [7]. It is known that on the theory side the CCFM physics is still to be completed. Below we outline the main points. For instance:

- the CCFM system of equations has been so far solved in decoupled approximation in the gluonic (for short the equation for gluon density is usually called CCFM) and non-singlet channels neglecting correlations between partons [8]. This might result in improper treatment of the kinematical region where the gluon degrees of freedom are less dominant than sea quarks. Gluon might be artificially dominating over quarks where it should actually be suppressed. Furthermore the corrections coming from inclusion of transversal momentum dependence to splitting functions $P_{g q}$ and $P_{q q}$ are not known and in order to have complete picture they should be calculated [8]. 


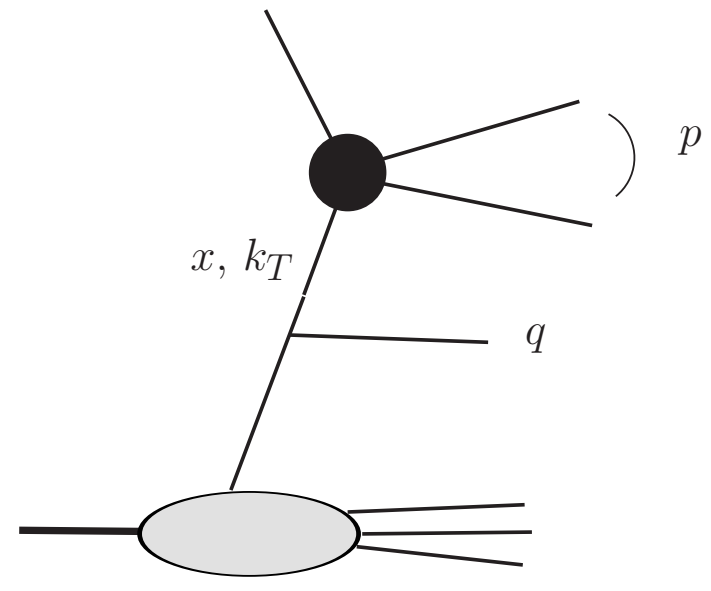

Figure 1. The plot visualizing kinematical variables used in the CCFM equation. The black blob represents hard process while the gray oval represents hadron.

- the impact of kinematical effects introducing energy conservation in the CCFM evolution have not been investigated in all detail. As it turns out from our study it is necessary to revisit the inclusion of the so called kinematical constraint [9] into the CCFM equation. The orgin of the kinematical constraint follows from refining the assumption that the $t$-channel off-shell gluon's 4 -momentum is dominated by $k_{T}^{2}$. The kinematical constraint causes suppression of the gluon density and even overrides the angular ordering in regions of large $k_{T}$.

- only recently the CCFM equation has been promoted to non-linear equation [10-12] therefore allowing for the possibility to investigate interplay of coherence effects and saturation [1] in exclusive processes like dijet production at the LHC [13-20]. In particular the important question is what is the role of the angular ordering and kinematical effect in the evolution at the non-linear level. The optimal form of initial conditions is also not known.

This publication is a continuation of the work done in $[21,22]$. We compare numerically forms of non-Sudakov form factor used in the literature and solve the full CCFM equation and its non-linear extension - including the $1 /(1-z)$ pole and kinematical the constraint in the kernel of the equation i.e. not only in the non-Sudakov form factor. However we keep $\alpha_{s}$ constant in order to have clear picture of the role of the kinematical effects.

\section{The CCFM equation}

The CCFM equation for gluon density reads:

$$
\begin{aligned}
\mathcal{A}\left(x, k_{T}, p\right)= & \mathcal{A}_{0}\left(x, k_{T}, p\right) \\
& +\bar{\alpha}_{S} \int \frac{d^{2} \overline{\mathbf{q}}}{\pi \overline{\mathbf{q}}^{2}} \int_{x}^{1-\frac{Q_{0}}{\bar{q}}} d z \theta(p-z \bar{q}) \mathcal{P}\left(z, k_{T}, \bar{q}\right) \Delta_{S}\left(p, z \bar{q}, Q_{0}\right) \mathcal{A}\left(x / z, k_{T}{ }^{\prime}, \bar{q}\right),
\end{aligned}
$$


where $k^{\prime}=|\mathbf{k}+(1-z) \overline{\mathbf{q}}|$ and the moduli of the two dimensional vectors transversal to the collision plane are denoted $|\mathbf{k}| \equiv k_{T},|\mathbf{q}| \equiv q_{T}, x$ is gluon's longitudinal momentum fraction and $\bar{\alpha}_{s}=N_{c} \alpha_{s} / \pi$. Also the rescaled momentum is introduced as $\bar{q}=|\overline{\mathbf{q}}|=q_{T} /(1-z)$.

For details of kinematical variables see figure 1 . the splitting function assumes the form:

$$
\mathcal{P}\left(z, k_{T}, \bar{q}\right)=\frac{\Delta_{N S}\left(z, k_{T}^{2}, \bar{q}\right)}{z}+\frac{1}{1-z} .
$$

The Sudakov form-factor evaluated in double logarithmic approximation reads:

$$
\Delta_{S}\left(p, z \bar{q}, Q_{0}\right)=\exp \left(-\int_{(z \overline{\mathbf{q}})^{2}}^{p^{2}} \frac{d q^{\prime 2}}{q^{\prime 2}} \int_{0}^{1-\frac{Q_{0}}{||^{\prime} \mid}} d z^{\prime} \frac{\bar{\alpha}_{S}}{1-z^{\prime}}\right) .
$$

The non-Sudakov form-factor regularizing $1 / z$ singularity in angular ordered region is:

$$
\Delta_{N S}\left(z, k_{T}, \bar{q}\right)=\exp \left(-\bar{\alpha}_{s} \int_{z}^{1} \frac{d z^{\prime}}{z^{\prime}} \int_{z^{2} \bar{q}^{2}}^{k^{2}} \frac{d^{2} q^{\prime}}{q^{2 \prime}}\right)=\exp \left(-\bar{\alpha}_{S} \ln \frac{1}{z} \ln \frac{k_{T}^{2}}{z \bar{q}^{2}}\right)
$$

\subsection{The kinematical constraint}

The integration over $\overline{\mathbf{q}}$ in the equation (2.1), although being constrained from below by the soft cut-off $Q_{0}$, is not constrained by at $z \ll 1$ an upper limit thus violating the energy-momentum conservation. Moreover in the low $x$ formalism one requires that in the denominator of the off-shell gluon propagator one keeps terms that obey $\left|k^{2}\right|=k_{T}^{2}$. In order to be consistent as argued in [4] the non-Sudakov form-factor should be accompanied by a kinematical constraint limiting the above integration over $\bar{q}$. In approximated form it reads

$$
k_{T}^{2}>z \bar{q}^{2} .
$$

After including it in the (2.4) we have:

$$
\Delta_{N S}\left(z, k_{T}, \bar{q}\right)=\exp \left(-\bar{\alpha}_{S} \ln \frac{1}{z} \ln \frac{k_{T}^{2}}{z \bar{q}^{2}} \theta\left(k_{T}^{2}-z \bar{q}^{2}\right)\right) .
$$

The condition (2.5) at $z \ll 1$ guaranties that $\left|k^{2}\right| \simeq k_{T}^{2}$. In [9] it has been extended to region including also the case when $z \sim 1$. Below we re-obtain the full form of kinematical constraint emphasizing its role in conservation of energy. Having $k=z p^{+}+\bar{z} p^{-}+k_{\perp}$ with $p^{+}$and $p^{-}$being the initial state gluon momenta + and - components, we can write the expression for $k^{2}$

$$
k^{2}=-z \bar{z} \hat{s}-k_{T}^{2},
$$

with $\hat{s}=\left(p^{+}+p^{-}\right)^{2}=2 p^{+} \cdot p^{-}$. Note, that for the full propagator $\left|k^{2}\right|>k_{T}^{2}$, thus the full propagator causes stronger suppression of the amplitude which has to be taken into account when using $\left|k^{2}\right| \simeq k_{T}^{2}$. A cut-off like (2.7) is a simple implementation of the suppression. The condition $\left|k^{2}\right| \simeq k_{T}^{2}$ translates approximately to

$$
k_{T}^{2}>z \bar{z} \hat{s} .
$$



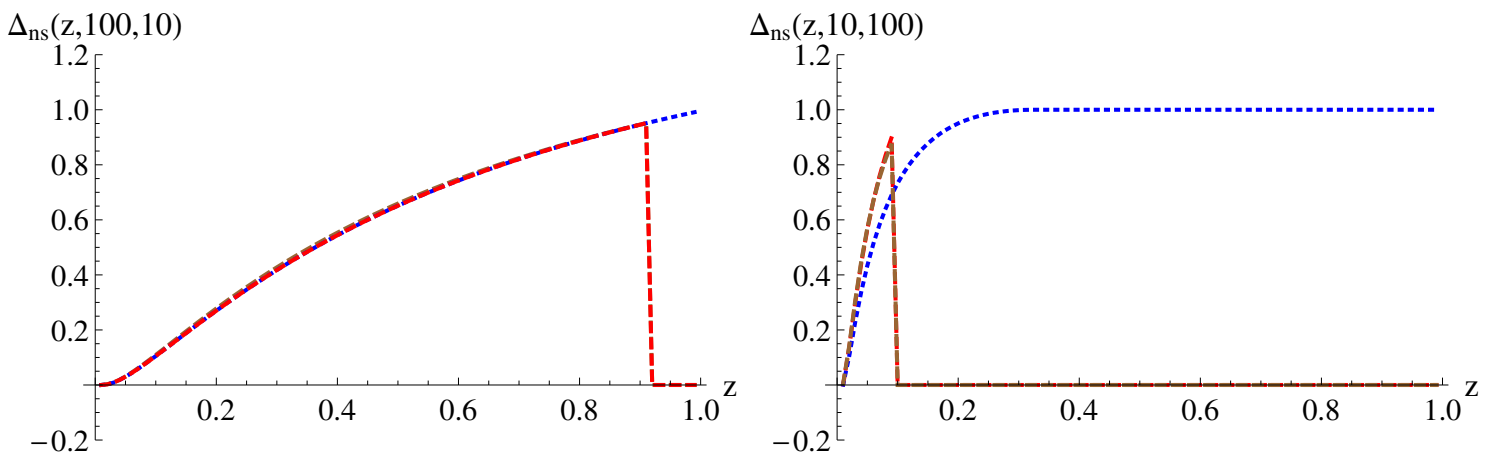

Figure 2. Plots visualize the various forms of non-Sudakov form factor. On the left we choose the situation when $k_{T}=10 \mathrm{GeV}$ and $\bar{q}=4 \mathrm{GeV}$ while on the right $k_{T}=4 \mathrm{GeV}$ and $\bar{q}=10 \mathrm{GeV}$. The red line (dashed long) corresponds to form-factor given by formula (2.6), the brown line (dashed medium) corresponds to form-factor given by formula (2.11) while blue line (dashed short) to form-factor given by formula (2.12). The form-factor given by (2.11) is multiplied by the function $\theta\left(k_{T} /(1-z) \bar{q}^{2}-z\right)$.

Using the identity $q^{2}=\bar{z}(1-z) \hat{s}-q_{T}^{2}=0$, we can express $\bar{z} \hat{s}=q_{T}^{2} /(1-z)$ and insert it into (2.8) to obtain:

$$
k_{T}^{2}>\frac{z q_{T}^{2}}{1-z}=z(1-z) \bar{q}^{2} .
$$

The lower bound on $z>x$ results in the upper bound on $q_{T}^{2}<k_{T}^{2} / x \simeq \hat{s}$ providing local condition for energy-momentum conservation. The CCFM with the kinematical constraint included reads [9]:

$$
\begin{aligned}
\mathcal{A}\left(x, k_{T}, p\right)= & \mathcal{A}_{0}\left(x, k_{T}, p\right)+\bar{\alpha}_{S} \int \frac{d^{2} \overline{\mathbf{q}}}{\pi \overline{\mathbf{q}}^{2}} \int_{x}^{1-\frac{Q_{0}}{|\overline{\mathbf{q}}|}} d z \theta\left(\frac{k_{T}^{2}}{(1-z) \bar{q}^{2}}-z\right) \\
& \times \theta(p-z|\bar{q}|) \mathcal{P}\left(z, k_{T}, \bar{q}\right) \Delta_{S}\left(p, z \bar{q}, Q_{0}\right) \mathcal{A}\left(x / z, k_{T}{ }^{\prime}, \bar{q}\right),
\end{aligned}
$$

and the non-Sudakov form-factor after inclusion of the full form of the kinematical constraint assumes the form

$$
\begin{array}{r}
\Delta_{N S}\left(z, k_{T}, \bar{q}\right)=\exp \left\{-\bar{\alpha}_{S} \int_{z}^{1} \frac{d z^{\prime}}{z^{\prime}} \Theta\left(\frac{\left(1-z^{\prime}\right) k_{T}^{2}}{(1-z)^{2} \bar{q}^{2}}-z^{\prime}\right) \times\right. \\
\left.\int \frac{d q^{\prime 2}}{q^{\prime 2}} \Theta\left(k_{T}^{2}-q^{\prime 2}\right) \Theta\left(q^{\prime}-z^{\prime} \bar{q}\right)\right\} .
\end{array}
$$

Please note the presence of the function $\theta\left(\frac{k_{T}^{2}}{(1-z) \bar{q}^{2}}-z\right)$. At the level of the kernel of the BFKL equation obtained directly from Feynmann diagrams (virtual and real corrections are kept apart) one imposes the kinematical constraint at the so called "unresummed" level on real emissions. However in [9] it has been observed, at a level of BFKL equation, that imposing the kinematical constraint on unresummed equation and performing algebraic transformations leading to resummed equation one obtains a $\theta$-function affecting the full kernel. The starting point of the CCFM is a resummed kernel, therefore this result 
suggests how the complete form of the kinematical constraint should be imposed on the kernel of CCFM. The authors of [9] solve the CCFM at small $z$ limit therefore the function $\theta\left(\frac{k_{T}^{2}}{(1-z) \bar{q}^{2}}-z\right)$ is neglected and in most of the phenomenological and theoretical applications of the CCFM this term is neglected [7, 15, 23-28] as well. The following form of non-Sudakov form factor is usually being used:

$$
\begin{aligned}
\Delta_{N S}\left(z, k_{T}, \bar{q}\right) & =\exp \left(-\bar{\alpha}_{S} \int_{z}^{z_{0}} \frac{d z^{\prime}}{z^{\prime}} \int \frac{d q^{\prime 2}}{q^{\prime 2}} \Theta\left(k^{2}-q^{\prime 2}\right) \Theta\left(q^{\prime}-z^{\prime} \bar{q}\right)\right) \\
& =\exp \left(-\bar{\alpha}_{S} \log \left(\frac{z_{0}}{z}\right) \log \left(\frac{k^{2}}{z_{0} z \bar{q}^{2}}\right)\right),
\end{aligned}
$$

where

$$
z_{0}= \begin{cases}1, & \text { if }\left(k_{T} / \bar{q}\right) \geq 1 \\ k_{T} / \bar{q}, & \text { if } z<\left(k_{T} / \bar{q}\right)<1 \\ z, & \text { if }\left(k_{T} / \bar{q}\right) \leq z\end{cases}
$$

and the discussed above $\theta$-function is not taken into account.

As shown in the figure 2 the simplified non-Sudakov form-factor (2.6) approximates well the exact one (2.11) in regions of $z$ where the $\theta$-function is not affecting the kernel while (2.12) is quite different. In our analysis we consider following equations:

$$
\begin{aligned}
\mathcal{A}\left(x, k_{T}, p\right)= & \mathcal{A}_{0}\left(x, k_{T}, p\right)+\bar{\alpha}_{S} \int \frac{d^{2} \overline{\mathbf{q}}}{\pi \overline{\mathbf{q}}^{2}} \int_{x}^{1-\frac{Q_{0}}{\bar{q}}} d z \theta\left(\frac{k_{T}^{2}}{(1-z) \bar{q}^{2}}-z\right) \\
& \times \theta(p-z \bar{q}) \mathcal{P}\left(z, k_{T}, \bar{q}\right) \Delta_{S}\left(p, z \bar{q}, Q_{0}\right) \mathcal{A}\left(x / z, k_{T}{ }^{\prime}, \bar{q}\right),
\end{aligned}
$$

where the splitting function includes non-Sudakov given by formula (2.11) and

$$
\begin{aligned}
\mathcal{A}\left(x, k_{T}, p\right)= & \mathcal{A}_{0}\left(x, k_{T}, p\right)+\bar{\alpha}_{S} \int \frac{d^{2} \overline{\mathbf{q}}}{\pi \overline{\mathbf{q}}^{2}} \int_{x}^{1-\frac{Q_{0}}{\bar{q}}} d z \\
& \times \theta(p-z \bar{q}) \mathcal{P}\left(z, k_{T}, \bar{q}\right) \Delta_{S}\left(p, z \bar{q}, Q_{0}\right) \mathcal{A}\left(x / z, k_{T}{ }^{\prime}, \bar{q}\right),
\end{aligned}
$$

where the non-Sudakov given by formula (2.12).

Note added in the proof reading. We were informed by Hannes Jung that the kinematical constraint (2.9) has been recently included in the CASCADE Monte Carlo.

\subsection{Saturation effects and kinematical constraint combined}

To account for gluon recombination at large gluon densities the CCFM equation has been promoted to non-linear equation by including a quadratic term [10-12] which reads:

$$
\begin{aligned}
\mathcal{A}\left(x, k_{T}, p\right)= & \mathcal{A}_{0}\left(x, k_{T}, p\right)+\bar{\alpha}_{S} \int \frac{d^{2} \overline{\mathbf{q}}}{\pi \overline{\mathbf{q}}^{2}} \int_{x}^{1-\frac{Q_{0}}{\bar{q}}} d z \theta\left(\frac{k_{T}^{2}}{(1-z) \bar{q}^{2}}-z\right) \theta(p-z \bar{q}) \mathcal{P}\left(z, k_{T}, \bar{q}\right) \\
& \times \Delta_{S}\left(p, z \bar{q}, Q_{0}\right)\left(\mathcal{A}\left(x / z, k_{T}^{\prime}, \bar{q}\right)-\delta\left(\bar{q}^{2}-\frac{k_{T}^{2}}{(1-z)^{2}}\right) \bar{q}^{2} \mathcal{A}^{2}(x / z, \bar{q}, \bar{q})\right), \quad(2.16)
\end{aligned}
$$


where we also included the kinematical constraint of the form (2.11) in the kernel. Simpler versions of the equation above have been already analyzed in [22] and it has been observed that

- the equation leads to phenomenon called saturation at the saturation scale $[22,26]$

- the saturation strongly suppresses the gluon density at low $x$ and low $k_{T}$.

The natural question arises: how are these results modified when some of the approximations are not taken and how are they modified if the kinematical effect is imposed in the full form?

\section{Numerical results and the discussion}

We present numerical results with $\bar{\alpha}_{S}=0.2$ and set the parameter $Q_{0}$ to $Q_{0}=1 \mathrm{GeV}$. To study the variation of the results depending on $Q_{0}$ we produce a solution also for $Q_{0}=0.5 \mathrm{GeV}$. The choices of the starting scale are motivated by a possibility to compare the equations in a region where both linear and non-linear equations are phenomenologically relevant.

\subsection{Linear equations}

We use an initial condition which includes resummed virtual and unresolved contributions, according to [22] and [27], in the form

$$
\mathcal{A}_{0}\left(x, k_{T}, p\right)=A \Delta_{R}\left(x, k_{T}, Q_{0}\right) \Delta_{S}\left(p, Q_{0}, Q_{0}\right) / k_{T},
$$

where $A=1 / 2$ and $\Delta_{R}\left(z, k_{T}, Q_{0}\right)=\exp \left(-\bar{\alpha}_{S} \log \frac{1}{z} \log \frac{k_{T}^{2}}{Q_{0}^{2}}\right)$ is the Regge form-factor.

The first observation we make from the plots in figures 3,5 and 6 is that the solutions of equations we study differ significantly. The solutions exhibit also similar features. Solutions of both versions of the kernel with kinematical constraint exhibit a local maximum as functions of $k_{T}$ and $p$ with $x$ and $p$ or $k_{T}$ correspondingly fixed. The positions of local maxima in the plots of $p$ dependence are correlated with the value of $k_{T}$, with a shift to higher $k_{T}$ for the solution of the equation (2.15). The peak can be explained by the fact that the contribution of the integral on the right hand side of (2.14) peaks at around $k_{T} \sim p$. To conclude: the peak is a result of presence of $\theta(p-z \bar{q})-$ i.e. angular ordering condition. Similar peaks are present also in the plots of $k_{T}$ dependence and resemble Sudakov suppression of $k_{T}$ scales of the order of $p$ in case of equation (2.14) [20]. However, in the case of (2.15) it seems, that the position of the peak does not depend on the value of $p$. It seems, that the kernel with the kinematical constraint included in an implicit way (2.15) produces similar effect, though weaker, as an implicit inclusion of the $\theta$-function only in the case of $p$ dependence, but for the $k_{T}$ dependence leads to much smaller suppression. Thus the peak observed in solution of (2.15) is 'buried' under the result of the evolution. We can conclude that the peak in the $k_{T}$ dependence is a result of an interplay of the inclusion of the explicit $\theta\left(\frac{k_{T}^{2}}{(1-z) \bar{q}^{2}}-z\right)$ factor and the Sudakov effect. 

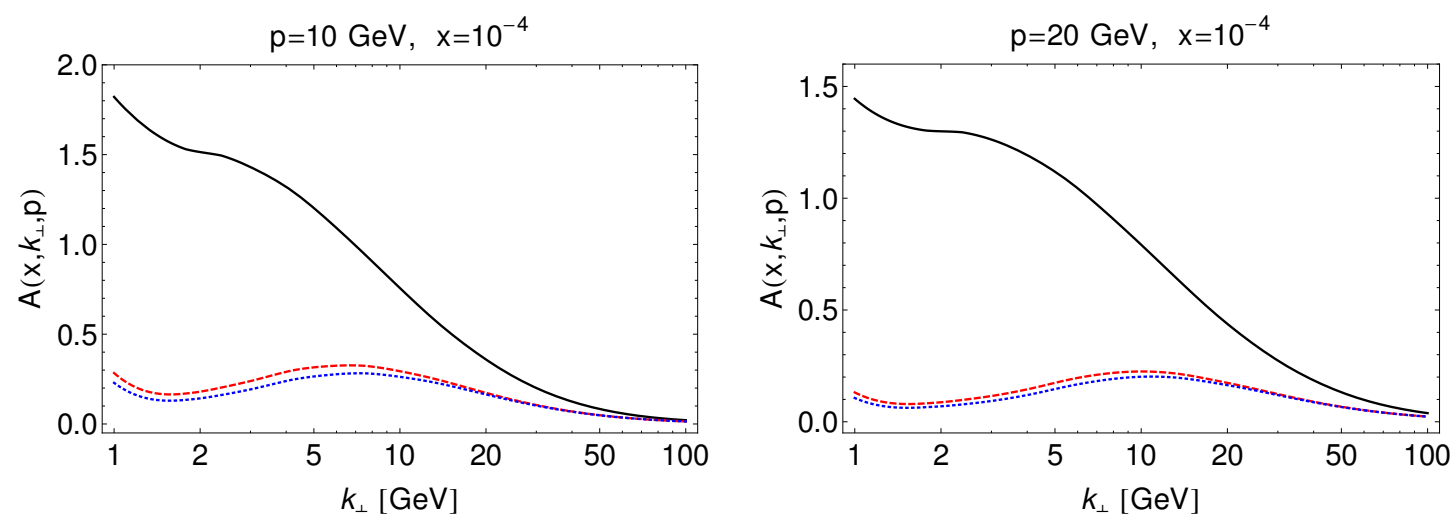

Figure 3. Red (dashed) - equation (2.14), black (solid) - equation (2.15), blue (dotted) - equation (2.16).
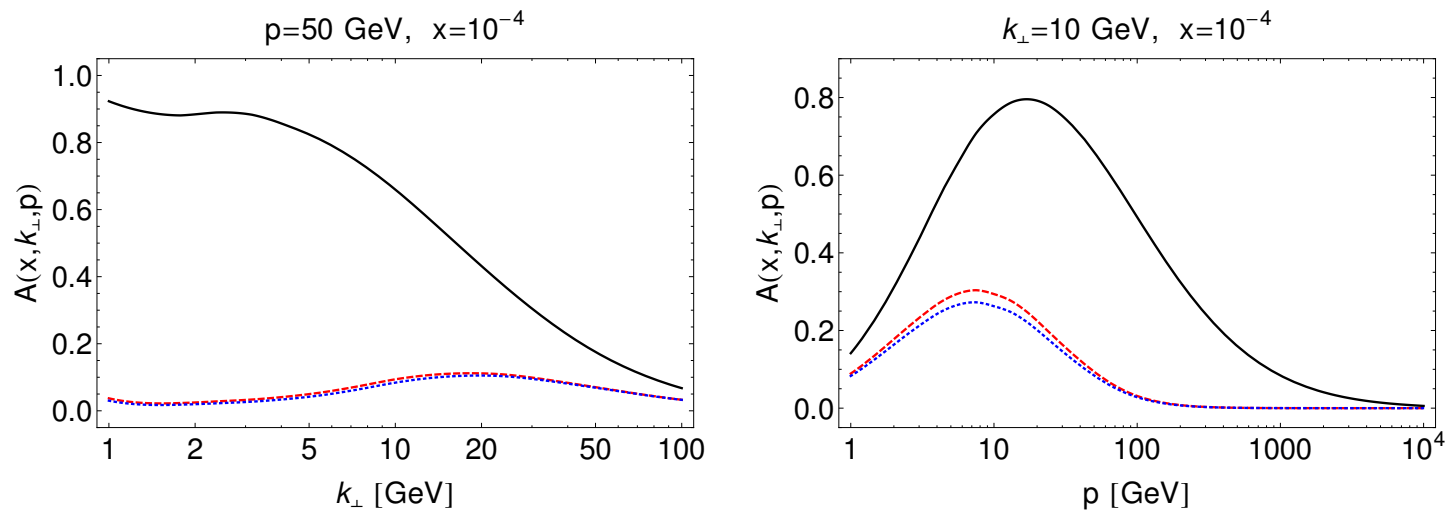

Figure 4. Red (dashed) - equation (2.14), black (solid) - equation (2.15), blue (dotted) - equation (2.16).
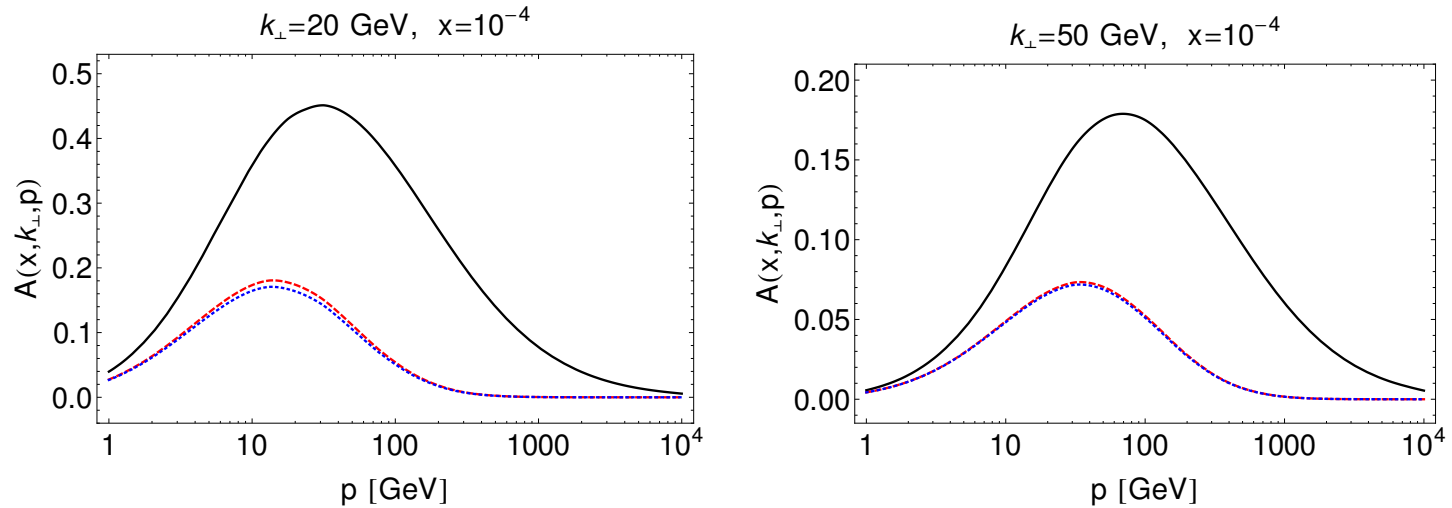

Figure 5. Red (dashed) - equation (2.14), black (solid) - equation (2.15), blue (dotted) - equation (2.16). 

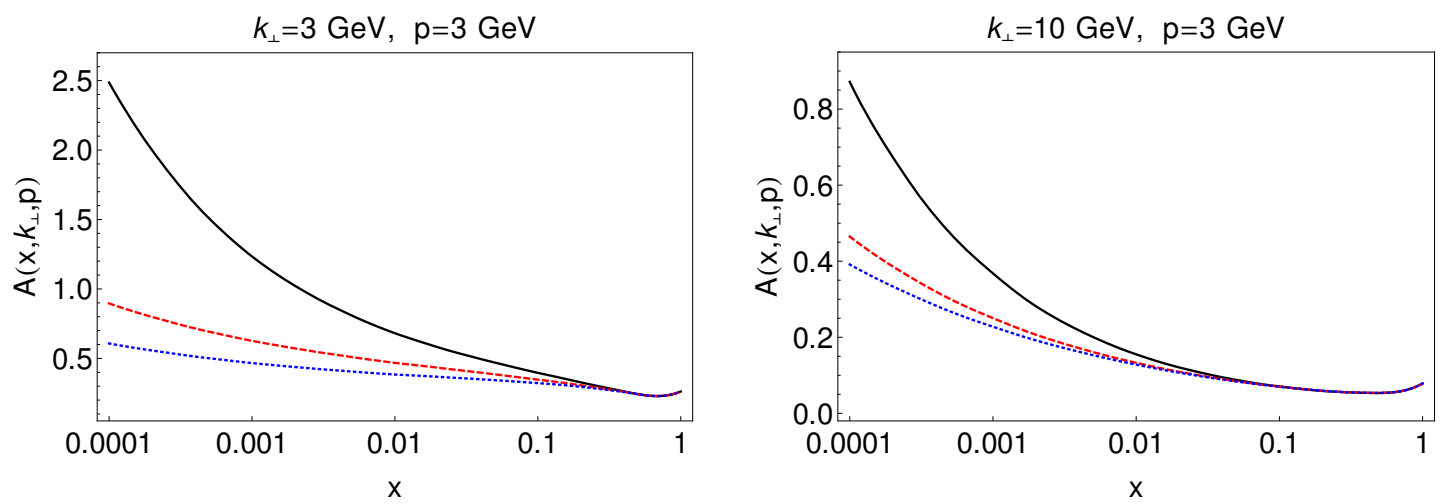

Figure 6. Red (dashed) - equation (2.14), black (solid) - equation (2.15), blue (dotted) - equation (2.16).

\subsection{Non-linear equation}

We set the parameter characterizing the strength of the non-linear term $R$ to the value $R=\sqrt{1 / \pi} \mathrm{GeV}$ in the equation (2.16).

By comparing the CCFM and KGBJS equations we see, that the equations give quite similar distributions. This effect (for our choice of the starting scale $Q_{0}$ ) comes from the fact, that the kinematical constraint suppresses the growth of the gluon so much, that the non-linear effects enter only at very low $x$. Observations made in previous paragraphs are confirmed in 2-dimensional plots (figures 7-8), where we plot relative difference of two amplitudes, solutions of the CCFM and the KGBJS equations, defined by the quantity

$$
\beta\left(x, k_{T}, p\right)=\frac{\left|\mathcal{A}_{C C F M}\left(x, k_{T}, p\right)-\mathcal{A}_{K G B J S}\left(x, k_{T}, p\right)\right|}{\mathcal{A}_{C C F M}\left(x, k_{T}, p\right)} .
$$

The function $\beta\left(x, k_{T}, p\right)$, introduced before in [22], can be used to measure the strength of the non-linear effects and to define a saturation scale using the condition:

$$
\beta\left(x, Q_{s}(x, p), p\right)=\text { const. }
$$

or $p$-related saturation scale:

$$
\beta\left(x, k_{T}, P_{s}\right)=\text { const. }
$$

The conditions above can be seen as equipotential lines in 2-dimensional plots in figures 7-8, where different equipotential lines correspond to different constants on the right-hand side of the equation above. The change in the slope of the $\beta\left(x, Q_{s}(x, p), p\right)$ at around $k_{T}=p$ reported in [22], apparent in figures $7-8$, can be understood in the context of the peak at $p \sim k_{T}$ (figures $3-4$ ). For $k_{T}>p$ the contribution of the integral on the right-hand side of the CCFM equation decreases and the gluon density is dominated by the initial condition.

By comparing the plots figures $7-8$ to analogous plots in [22] we see that their main features are very similar. We therefore conclude that the low $-x$ approximation of the KGBJS and CCFM equations taken in [22] does not, at least, modify the relative difference between linear and non-linear equation since the modifications of the kernel did not spoil the saturation pattern visible in the full equation. 

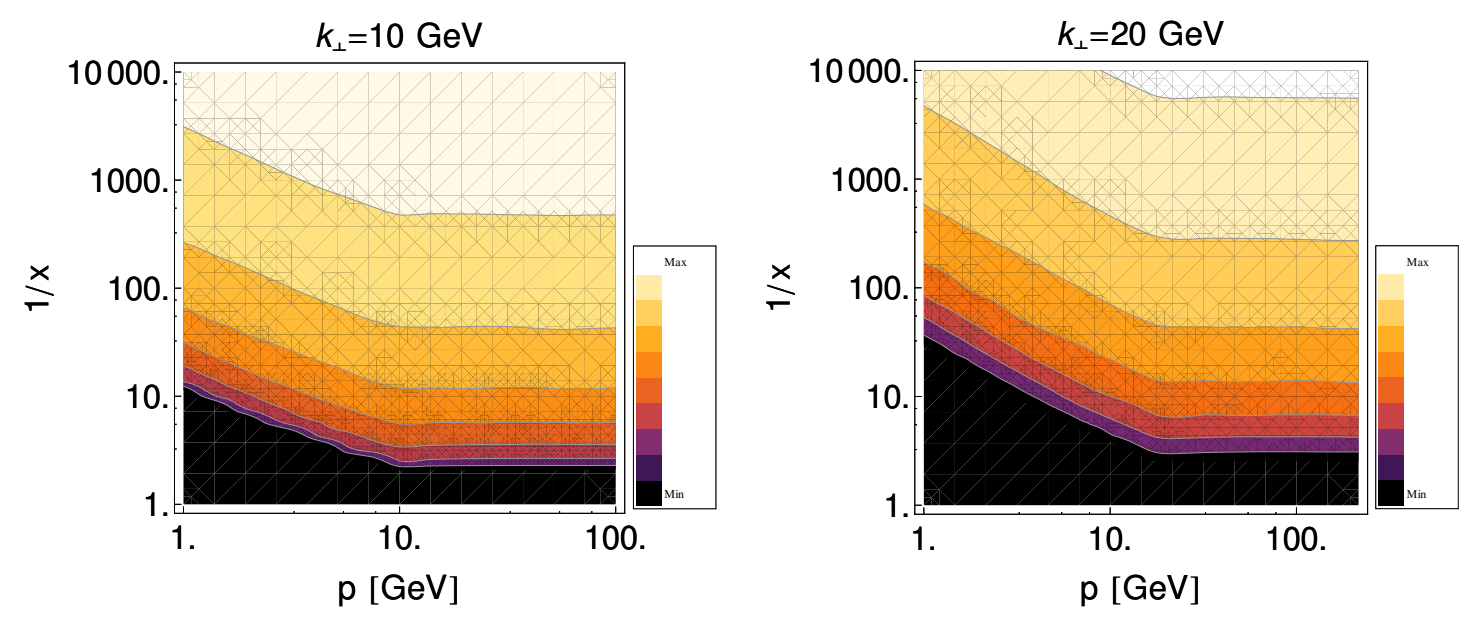

Figure 7. Relative ratio of CCFM (2.14) and KGBJS (2.16) solutions. Distributions with definite $k_{T}$ for varying value of $p$.
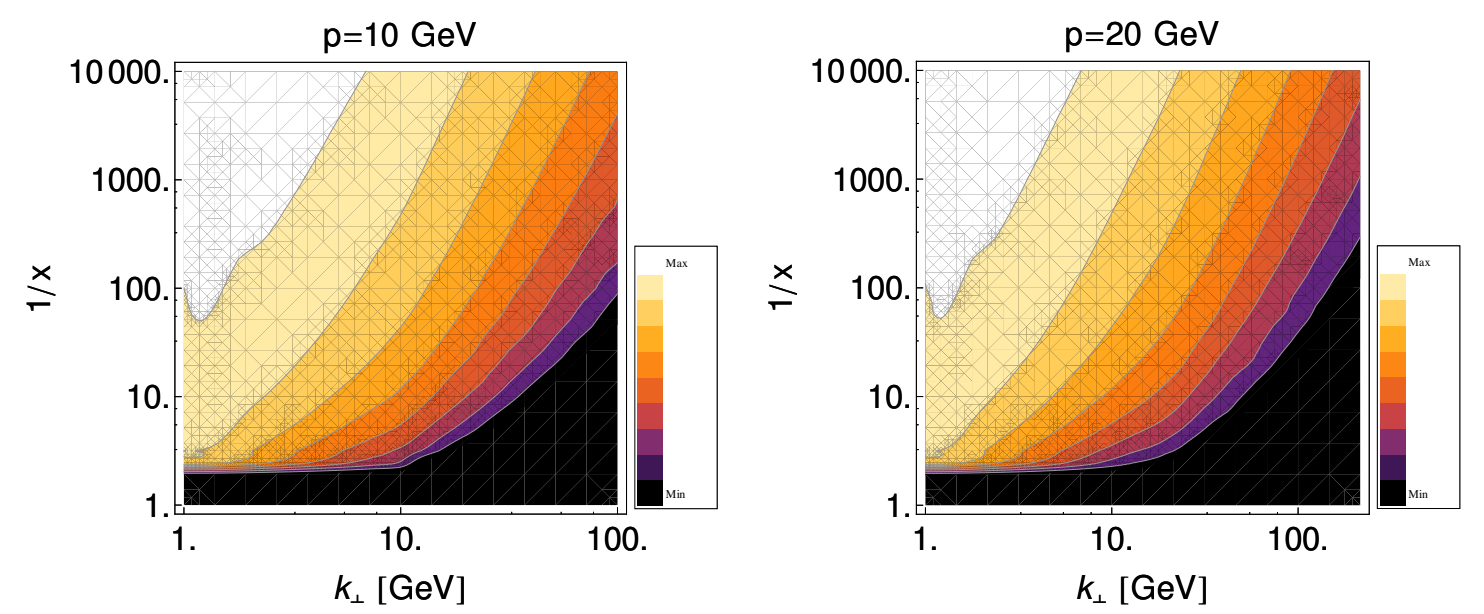

Figure 8. Relative ratio of CCFM (2.14) and KGBJS (2.16) solutions. Distributions with definite $p$ for varying value of $k_{T}$.

\subsection{Dependence on the starting scale}

The starting scale $Q_{0}$ dependence is studied in figures 9-10 using equations (2.14) and (2.16). We observe variation from few up to $25 \%$ mostly in the local maximum of the $k_{T}$ and $p$ distributions. There is a peculiarity of the dependence on $Q_{0}$ of the $k_{T}$ distributions near the boundary $k_{T}=Q_{0}$ which can be explained by the dependence on the initial condition on the parameter $Q_{0}$. Near the point $k_{T}=Q_{0}$ the initial condition dominates and since it depends on the parameter the solutions also depend strongly on this parameter. We can tell, though, that for $k_{T} \gg p$ and $p \gg k_{T}$ the difference diminishes. 

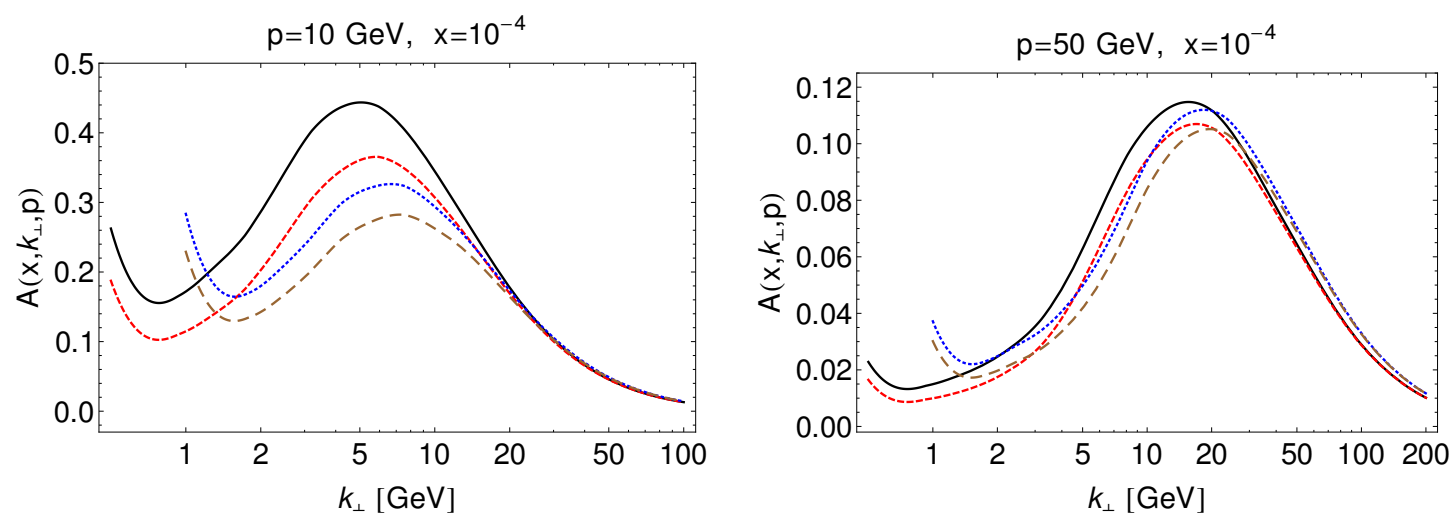

Figure 9. Solutions compared with $Q_{0}=0.5 \mathrm{GeV}$ and $Q_{0}=1 \mathrm{GeV}$. Black solid - linear CCFM with $Q_{0}=0.5 \mathrm{GeV}$, red dashed - non-linear CCFM with $Q_{0}=0.5 \mathrm{GeV}$, blue dotted - linear CCFM with $Q_{0}=1 \mathrm{GeV}$, brown dashed longer - linear CCFM with $Q_{0}=1 \mathrm{GeV}$.
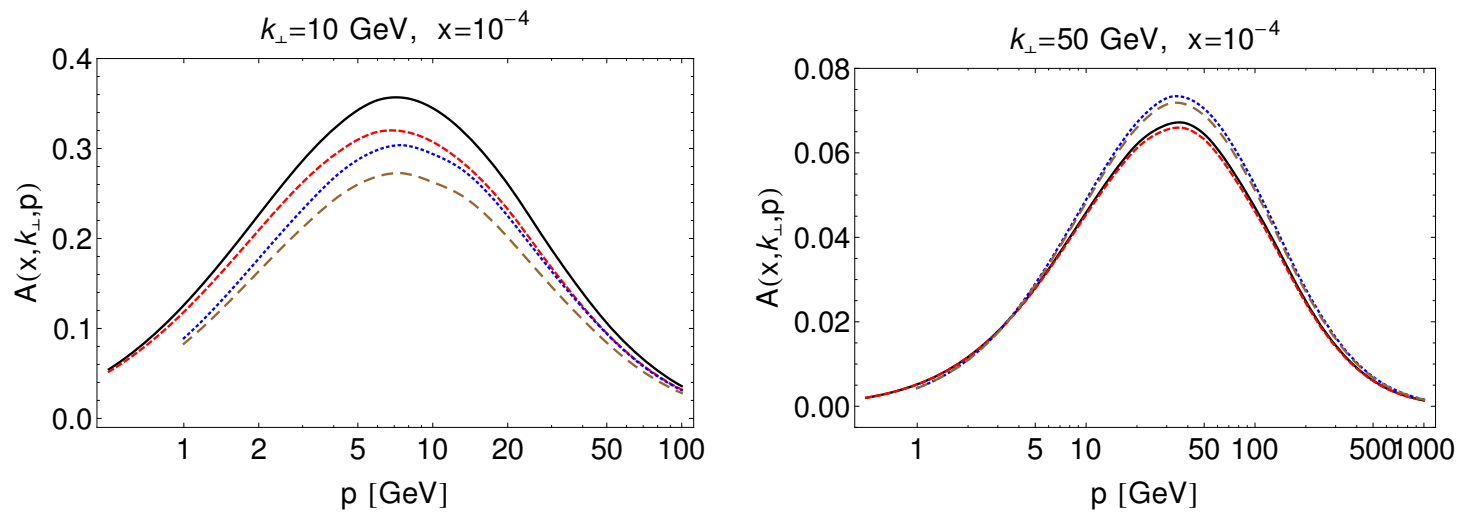

Figure 10. Solutions compared with $Q_{0}=0.5 \mathrm{GeV}$ and $Q_{0}=1 \mathrm{GeV}$. Black solid - linear CCFM with $Q_{0}=0.5 \mathrm{GeV}$, red dashed - non-linear CCFM with $Q_{0}=0.5 \mathrm{GeV}$, blue dotted - linear CCFM with $Q_{0}=1 \mathrm{GeV}$, brown dashed longer - linear CCFM with $Q_{0}=1 \mathrm{GeV}$.

\section{Conclusions and outlook}

We have solved linear and non-linear version of the CCFM equation with the full kernel, constant $\alpha_{s}$ and kinematical constraint included in two different ways.

Our numerical results make us conclude that the inclusion of the kinematical constraint in its full form into the CCFM equation seems to affect largely the distribution of gluons. In particular inclusion of the kinematical constraint without omitting the $\theta$-function in the kernel of the equation causes strong suppression of the solution for all values of transversal momenta $k_{T}$. The effect is stronger than the implicit kinematical constraint included via modification of the non-Sudakov form-factor widely used in literature. We expect that the suppression of the distribution will have significant effects on description of decorelations in azimuthal angle of forward-central jets $[15,29]$. In particular we expect that the inclusion of the $\theta$-function is crucial for suppression of the cross section of emission of gluons in small angles since this configuration corresponds to large values of $k_{T}$. However, the conclusive 
comparison of the different approaches presented in this article can be done only after fitting the initial conditions of the considered equations to data, this we, however, leave for future tasks.

\section{Acknowledgments}

We would like to acknowledge useful discussions with Dawid Toton who participated in the project in the early stage. The research of Krzysztof Kutak is supported by NCN grant DEC-2013/10/E/ST2/00656. Michal Deak acknowledges support from Juan de la Cierva programme (JCI-2011-11382). This work has been supported in part by the Spanish Government and ERDF funds from the EU Commission [Grants No. FPA2011-23778, FPA2014-53631-C2-1-P No. CSD2007-00042 (Consolider Project CPAN)].

Open Access. This article is distributed under the terms of the Creative Commons Attribution License (CC-BY 4.0), which permits any use, distribution and reproduction in any medium, provided the original author(s) and source are credited.

\section{References}

[1] L.V. Gribov, E.M. Levin and M.G. Ryskin, Semihard Processes in QCD, Phys. Rept. 100 (1983) 1 [INSPIRE].

[2] S. Catani, M. Ciafaloni and F. Hautmann, High-energy factorization and small-x heavy flavor production, Nucl. Phys. B 366 (1991) 135 [inSPIRE].

[3] M. Ciafaloni, Coherence Effects in Initial Jets at Small $Q^{2} / s$, Nucl. Phys. B 296 (1988) 49 [INSPIRE].

[4] S. Catani, F. Fiorani and G. Marchesini, Small-x Behavior of Initial State Radiation in Perturbative QCD, Nucl. Phys. B 336 (1990) 18 [inSPIRE].

[5] S. Catani, F. Fiorani and G. Marchesini, QCD Coherence in Initial State Radiation, Phys. Lett. B 234 (1990) 339 [INSPIRE].

[6] H. Jung et al., The CCFM Monte Carlo generator CASCADE version 2.2.03, Eur. Phys. J. C 70 (2010) 1237 [arXiv: 1008.0152] [InSPIRE].

[7] F. Hautmann and H. Jung, Transverse momentum dependent gluon density from DIS precision data, Nucl. Phys. B 883 (2014) 1 [arXiv:1312.7875] [INSPIRE].

[8] F. Hautmann, H. Jung and S.T. Monfared, The CCFM uPDF evolution uPDFevolv Version 1.0.00, Eur. Phys. J. C 74 (2014) 3082 [arXiv:1407.5935] [INSPIRE].

[9] J. Kwiecinski, A.D. Martin and P.J. Sutton, Constraints on gluon evolution at small $x, Z$. Phys. C 71 (1996) 585 [hep-ph/9602320] [INSPIRE].

[10] K. Kutak, K. Golec-Biernat, S. Jadach and M. Skrzypek, Nonlinear equation for coherent gluon emission, JHEP 02 (2012) 117 [arXiv:1111.6928] [INSPIRE].

[11] K. Kutak, Nonlinear extension of the CCFM equation, arXiv:1206.1223 [INSPIRE].

[12] K. Kutak, Resummation in nonlinear equation for high energy factorisable gluon density and its extension to include coherence, JHEP 12 (2012) 033 [arXiv:1206.5757] [INSPIRE]. 
[13] M. Deak, F. Hautmann, H. Jung and K. Kutak, Forward Jet Production at the Large Hadron Collider, JHEP 09 (2009) 121 [arXiv:0908.0538] [INSPIRE].

[14] M. Deak, F. Hautmann, H. Jung and K. Kutak, Jets in the forward region at the LHC, arXiv:0908.1870 [INSPIRE].

[15] M. Deak, F. Hautmann, H. Jung and K. Kutak, Forward-Central Jet Correlations at the Large Hadron Collider, arXiv: 1012.6037 [INSPIRE].

[16] K. Kutak and S. Sapeta, Gluon saturation in dijet production in $p$-Pb collisions at Large Hadron Collider, Phys. Rev. D 86 (2012) 094043 [arXiv: 1205.5035] [INSPIRE].

[17] CMS collaboration, Measurement of the inclusive production cross sections for forward jets and for dijet events with one forward and one central jet in pp collisions at $\sqrt{s}=7 \mathrm{TeV}$, JHEP 06 (2012) 036 [arXiv: 1202.0704] [INSPIRE].

[18] A. van Hameren, P. Kotko, K. Kutak and S. Sapeta, Small-x dynamics in forward-central dijet decorrelations at the LHC, Phys. Lett. B 737 (2014) 335 [arXiv:1404.6204] [INSPIRE].

[19] A. van Hameren, P. Kotko, K. Kutak, C. Marquet and S. Sapeta, Saturation effects in forward-forward dijet production in p + Pb collisions, Phys. Rev. D 89 (2014) 094014 [arXiv: 1402.5065] [INSPIRE].

[20] K. Kutak, Hard scale dependent gluon density, saturation and forward-forward dijet production at the LHC, Phys. Rev. D 91 (2015) 034021 [arXiv: 1409.3822] [INSPIRE].

[21] M. Deak, Estimation of saturation and coherence effects in the KGBJS equation - a non-linear CCFM equation, JHEP 07 (2013) 087 [arXiv:1209.6092] [INSPIRE].

[22] K. Kutak and D. Toton, Gluon saturation scale from the KGBJS equation, JHEP 11 (2013) 082 [arXiv: 1306.3369] [INSPIRE].

[23] G.P. Salam, Associated quantities from the CCFM approach, in Brussels 1998, Deep inelastic scattering and QCD, pg. 543-547 hep-ph/9805322 [INSPIRE].

[24] G. Bottazzi, G. Marchesini, G.P. Salam and M. Scorletti, Small-x one particle inclusive quantities in the CCFM approach, JHEP 12 (1998) 011 [hep-ph/9810546] [INSPIRE].

[25] G.P. Salam, Soft emissions and the equivalence of BFKL and CCFM final states, JHEP 03 (1999) 009 [hep-ph/9902324] [INSPIRE].

[26] E. Avsar and A.M. Stasto, Non-linear evolution in CCFM: The interplay between coherence and saturation, JHEP 06 (2010) 112 [arXiv: 1005.5153] [INSPIRE].

[27] A. Bacchetta, H. Jung, A. Knutsson, K. Kutak and F. Samson-Himmelstjerna, A method for tuning parameters of Monte Carlo generators and a its application to the determination of the unintegrated gluon density, Eur. Phys. J. C 70 (2010) 503 [arXiv:1001.4675] [INSPIRE].

[28] G. Chachamis, M. Deak, A.S. Vera and P. Stephens, A comparative study of small x Monte Carlos with and without QCD coherence effects, Nucl. Phys. B 849 (2011) 28 [arXiv: 1102.1890] [INSPIRE].

[29] CMS collaboration, Measurement of azimuthal correlations between forward and central jets in proton proton collisions at $\sqrt{s}=7 \mathrm{TeV}$, CMS-PAS-FSQ-12-008. 